\title{
COMPARATIVO DE RENDIMIENTO E INCIDENCIA DE Alternaria solani EN SEIS ECOTIPOS DE COCONA (Solanum sessiliflorum)
}

\author{
Elsa OSPINO ${ }^{1}$, Luz BALCÁZAR ${ }^{2}$, Richard REMUZGO ${ }^{3}$ \\ 1 Universidad Nacional Agraria de la selva UNAS. Tingo María, Perú. Tesista de la Facultad de Ciencias Agrarias. \\ 2 Instituto de Investigaciones de la Amazonía Peruana, IIAP. Programa de biodiversidad Amazónica (PIBA). Jr. Piura 1076. \\ Docente Asociado, adscrito a la Facultad de Agronomía de La Universidad Nacional Agraria de la Selva. \\ Ibalcazar@iiap.org.pe \\ 3 Instituto de Investigaciones de la Amazonía Peruana, IIAP. Programa de Biodiversidad Amazónica (PIBA). Jr. Piura 1076.
}

\section{RESUMEN}

Se evaluaron el rendimiento y el número total de frutos de cocona (Solanum sessiliflorum) y la incidencia del hongo Alternaria solani en seis ecotipos de cocona: SH4, AMG2, CPK-3, KIJ101; SM y NE. Cada ecotipo constituyó un tratamiento distribuido en bloques completos al azar con tres repeticiones, la diferencia de medias se determinó por la prueba de Duncan $\alpha=0.05$. Los resultados muestran que el ecotipo CPK-3 con $27.13 \mathrm{tha}^{-1}$, es semejante al ecotipo AMG-2 con $20.87 \mathrm{tha}^{-1}$, ambos estadísticamente superiores a los ecotipos: SM, SH-4, NE y RIJ-101 con $15.79 \mathrm{t} \mathrm{ha}^{-1}, 13.00 \mathrm{t} \mathrm{ha}^{-1}, 11.54 \mathrm{t} \mathrm{ha}^{-1}$ y $9.97 \mathrm{t} \mathrm{ha}^{-1}$, respectivamente. El ecotipo CPK-3, con 126.37 frutos por planta, superó estadísticamente al ecotipo SM, con 26.28 frutos por planta. Con respecto a la incidencia de Alternaria solani a nivel de frutos, el ecotipo que alcanzó menor incidencia fue CPK-3, con $17.60 \%$, alcanzando la más alta tolerancia a la enfermedad. Los ecotipos con mayor rendimiento en este trabajo fueron los que poseían frutos de tamaño intermedio y con menor porcentaje de incidencia a Alternaria solani, como es el caso del ecotipo CPK-3(17.60\%).

PALABRAS CLAVE: Cocona, ecotipos, rendimiento, enfermedad, incidencia, frutales amazónicos.

\section{YIELD COMPARATIVE AND INCIDENCE OF Alternaria solani IN SIX ECOTYPES OF COCONA (Solanum sessiliflorum Dunal)}

\begin{abstract}
The Cocona's (Solanum sessiliflorum) yield and number of fruits and incidence of Alternaria solani were evaluated in six ecotypes of cocona: SH4, AMG2, CPK-3, RIJ101, SM and NE. A completely randomized block statistical design was used, distributed in six treatments with three blocks each, means difference was determined by Duncan $\alpha=0.05$. The evaluated parameters were yield and total number of fruits. Yield's results shows Ecotype CPK-3 has a performance equal to 27.13 tha-1, statistically similar to Ecotype AMG-2 which presents 20.87 tha-1. Ecotypes SM (15.79 tha-1), SH-4 (13.00 tha-1), NE (11.54 tha-1), and RIJ-101 (9.97 t ha-1) showed, statistically, a lower yield. Results of performance shows Ecotype CPK-3 has a performance equal to $27.13 \mathrm{t}$ ha-1, statistically similar to Ecotype AMG-2 which presents $20.87 \mathrm{t}$ ha- 1 . Ecotypes SM (15.79 t ha-1), SH-4 (13.00 t ha-1), NE (11.54 tha-1), and RIJ-101 (9.97 t ha-1) showed, statistically, a lower production. Results of number of fruits show Ecotype CPK-3 with 126.37 fruits per plant statistically superior to Ecotype SM (26.28 fruits per plant). Concerning the incidence of Alternaria solani at a fruit level, Ecotype CPK-3 showed the lowest incidence (17.6\%), placing this one as the most tolerant ecotype. In this paperwork, ecotypes with the best performance showed average fruit size and the lowest incidence of Alternaria solani at a fruit level, just like ecotype CPK-3.
\end{abstract}

KEYWORDS: Ecotypes, performance, disease incidence. 


\section{INTRODUCCIÓN}

La cocona es un cultivo que tiene buena adaptación al clima y al suelo predominante en la selva del Alto Huallaga. Esta especie tiene precocidad productiva, alto rendimiento y producción no estacional que permite cosechas permanentes, con buenas perspectivas económicas, por su consumo popular en estado natural y como valor agregado en distintas presentaciones: ensaladas, néctares, encurtidos y refrescos. La cocona es una especie nativa de ceja de selva y selva alta de América Tropical, que se distribuye naturalmente entre los 200 y 1,000 metros de altura en Colombia, Perú, Ecuador y Venezuela (Carbajal \& Balcázar, 2000).

Las condiciones ideales para el cultivo de la cocona se encuentran en las zonas de clima templado, con temperatura promedio anual de $17^{\circ} \mathrm{C}$ a $30^{\circ} \mathrm{C}$, sin presencia de heladas, con precipitación pluvial promedio de $1,500 \mathrm{~mm}$ a $4,500 \mathrm{~mm}$ por año, bien distribuidas, con humedad relativa de $70 \%$ a $90 \%$ y altitudes variables desde el nivel del mar hasta los $1,200 \mathrm{msnm}$. Se cultiva en diferentes tipos de suelo, preferentemente en los de textura arcillosa a franca, ricos en materia orgánica y con buen drenaje. Los ecotipos que tienen frutos pequeños toleran los suelos arcillosos y resisten mejor las enfermedades. Los ecotipos de frutos más grandes e intermedios son más exigentes con los suelos y mucho más sensibles a las enfermedades. Los rendimientos varían de $6 \mathrm{t} \mathrm{ha}^{-1}$ a $17 \mathrm{t} \mathrm{ha}^{-1}$, dependiendo del ecotipo. El número de frutos que produce una planta está en relación al tamaño del fruto. Las plantas con frutos pequeños ( $25 \mathrm{~g}$ a $40 \mathrm{~g}$ ) producen entre 87 y 119 frutos por planta, las que tienen frutos medianos ( $40 \mathrm{~g} \mathrm{a} 60 \mathrm{~g}$ ) producen entre 83 y 95 frutos por planta, aquellas con frutos grandes ( $141 \mathrm{~g}$ a 215 g) producen entre 39 y 24 frutos por planta (Villachica, 1996).

Los rendimientos obtenidos en diferentes trabajos de investigación entre varios ecotipos variaron desde $13 \mathrm{t} \mathrm{ha}^{-1}$, hasta $21 \mathrm{t} \mathrm{ha}^{-1}$, en una misma localidad y en diferentes localidades de la zona del Alto Huallaga. Balcázar et al (2011) obtuvieron $13 \mathrm{t}$ $\mathrm{ha}^{-1}$ de frutos de cocona sembrada a un distanciamiento de $2 \mathrm{~m}$ entre filas y $1.50 \mathrm{~m}$ entre plantas, con una densidad de 3,333 plantas por hectárea.

Robles (2008), al evaluar el rendimiento de cuatro ecotipos en Tingo María (TR, T2, CSA 101, SRN9), encontró que el ecotipo TR obtuvo mayor rendimiento con $44.11 \mathrm{t} \mathrm{ha}^{-1}$, seguido de los ecotipos T2, con $38.28 \mathrm{tha}^{-1}$ y CSA 101 con $19.82 \mathrm{t}$ $\mathrm{ha}^{-1}$. En cuanto al peso de fruto, el ecotipo TR fue superior con $221.01 \mathrm{~g}$, frente a los ecotipos $\mathrm{T} 2$ con 172.38 g $\quad$ y CSA 101 con 43.19 g respectivamente.
Asimismo, se promueve su consumo a nivel nacional e internacional (PROMPERU 2013), debido a sus bondades medicinales, ya que ayuda a reducir el colesterol y los triglicéridos (María, 2004). La enfermedad más agresiva que afecta a la cocona es causada por el hongo Alternaria solani Sorauer (Balcázar et.al., 2011). Denominada como "tizón" o "alternariosis" de la cocona, afecta a diferentes órganos de la planta y se considera como una enfermedad muy importante por las pérdidas económicas que produce en este cultivo (Balcázar et.al., 2011). El objetivo de la investigación fue determinar el rendimiento y la incidencia de la enfermedad causada por el hongo Alternaria solani en seis ecotipos de cocona.

\section{MATERIAL Y MÉTODOS}

El experimento se realizó en el Centro de Investigación y Producción de Tulumayo de la Universidad Nacional Agraria de la Selva, región Huánuco, 9 $9^{\circ} 17^{\prime} 58^{\prime \prime}$ latitud sur, 67 $10^{\prime} 07^{\prime}$ longitud oeste, a una altitud de $610 \mathrm{msnm}$. Los registros meteorológicos obtenidos durante el experimento en la estación meteorológica de Tulumayo, mostraron una precipitación total de $2,030 \mathrm{~mm}$, variando entre $38 \mathrm{~mm}$ a $450 \mathrm{~mm}$, la temperatura varió entre $24.9{ }^{\circ} \mathrm{C}$ y $26.15^{\circ} \mathrm{C}$ y la humedad promedio alcanzó el $83.13 \%$. La muestra de suelo obtenida presentó una textura francoarcillo-limosa, pH (6.70) de reacción neutra, porcentajes medios de materia orgánica $(3.2 \%)$ y nitrógeno total $(0.14 \%)$, contenido medio de fósforo $\left(14 \mu \mathrm{g} \mathrm{g}^{-1}\right)$ y potasio $\left(339 \mathrm{~kg} \mathrm{~K} 2 \mathrm{O} \mathrm{ha}^{-1}\right)$ y baja capacidad de intercambio catiónico $\left(6.15 \mathrm{cmol} \mathrm{kg}^{-1}\right)$. Los tratamientos representaron a seis ecotipos: $\mathrm{SH}-4$ (cocona Shambillo-Ucayali), AMG-2 (cocona Aramango-Amazonas), CPK-3 (cocona PichanakiJunín), KIJ-101 (cocona Rioja-Amazonas), SM (cocona san Martín) y NE (cocona Nueva esperanzaSan Martín).

\section{DISEÑO EXPERIMENTAL}

Se utilizó el diseño estadístico de bloque completamente al azar con seis tratamientos y tres repeticiones. Las características evaluadas de cada uno de los tratamientos fueron sometidas al análisis de variancia y prueba de significación estadística Duncan $\alpha=0.05$. El campo experimental presentó una dimensión de $2,125 \mathrm{~m}^{2}$, distribuidos en tres bloques de seis parcelas cada uno. Cada parcela tenía un área de de $90 \mathrm{~m}^{2}$ y un distanciamiento entre fila de $2 \mathrm{~m}$ y $1.5 \mathrm{~m}$ entre planta. El área de la parcela neta fue de $36 \mathrm{~m}^{2}$, con un total de 12 plantas evaluables.

Se realizaron seis cosechas, los frutos cosechados 
presentaron características típicas de madurez fisiológica. El rendimiento por hectárea se determinó mediante la producción en $\mathrm{t}_{\text {ha }}{ }^{-1}$; por el peso de frutos $(\mathrm{kg})$ por planta por el número de plantas por hectárea y por el peso de frutos por planta; y por último el peso del fruto por el número de frutos por planta $(\mathrm{Kg})$.

La incidencia (\%) se determinó contando el número de frutos enfermos y sanos obtenidos de plantas de la parcela neta en cada cosecha. Los frutos enfermos se identificaron a partir de los síntomas característicos de la enfermedad y el porcentaje de incidencia se determinó utilizando la fórmula de incidencia Anculle (1999).

El análisis de regresión y correlación se hizo considerando la variable independiente (X), los componentes de rendimiento integrado por caracteres productivos y la incidencia de Alternaria solani y la variable dependiente $(\mathrm{Y})$ integrada por el rendimiento (Di Rienzo, 2005).

\section{DETERMINACIÓN DE LOS CARACTERES EN EVALUACIÓN}

Los datos obtenidos se basaron en mediciones de los caracteres productivos de rendimiento: producción en toneladas por hectárea $\left(\mathrm{t} \mathrm{ha}^{-1}\right)$, peso de los frutos (kilogramos por planta), peso de frutos (g) por número de frutos por planta e incidencia de Alternaria solani utilizando la fórmula de incidencia propuesta por Anculle (1999).

Incidencia $(\%)=\left(\frac{\mathrm{N}^{\mathrm{o}} \text { de frutos enfermos }}{\mathrm{N}^{\circ} \text { total de frutos sanos }}\right) \times 100$

\section{RESULTADOS Y DISCUSIÓN}

EFECTO EN EL RENDIMIENTO TOTAL POR HECTÁREA.

El ecotipo CPK-3 alcanzó el mayor rendimiento con $27.13 \mathrm{t} \mathrm{ha}^{-1}$, seguido del ecotipo AMG-2, con 20.86 $\mathrm{t} \mathrm{ha}^{-1}$. Estos dos ecotipos no presentaron diferencias estadísticas significativas entre sí. El ecotipo CPK-3 presentó diferencias significativas con respecto a los otros ecotipos. Le siguieron en significancia los ecotipos SM, SH-4, NE y RIJ-110 (Tabla 1).

Se puede atribuir el comportamiento de los ecotipos de mayor rendimiento a sus características genéticas de amplio rango de adaptabilidad a la zona de estudio, mostrándose con mayor número y peso de frutos por planta. Un ecotipo puede tener frutos largos de buen diámetro, característica que conlleva una ventaja para acumular peso por fruto, pero que no es suficiente para alcanzar mayor rendimiento, ya que el rendimiento está dado por el peso y el número de frutos por planta (VILLACHICA, 1996; CARBAJAL y BALCAZAR, 2000). Debido a esto, el ecotipo CPK-3 alcanzó el mayor rendimiento, a pesar de poseer frutos con un peso de $34 \%$, hasta casi el $50 \%$, del peso de los ecotipos de frutos grandes: RIJ-101, SM y AMG-2 (CARBAJAL y BALCAZAR, 2000). Sin embargo, el rendimiento alcanzado por el ecotipo CPK-3 no supera a los ecotipos mostrados a continuación y estudiados en la misma zona: el N4 con 37.44 tha $^{-1}$, el N3 con 36.61 tha ${ }^{-1}$, el T6 con 35.10 tha $^{-1}$, el T4 con 34.16 tha $^{-1}$, el $\mathrm{N} 1$ con $31.14 \mathrm{tha}^{-1}$, el R2 con 30.26 tha $^{-1}$ (GÓMEZ, 1997) y el TR con $44.12 \mathrm{t} \mathrm{ha}^{-1}$ (ROBLES, 2008). Estos resultados se deben a que estos ecotipos estudiados poseían frutos más grandes, con un intervalo de largo de fruto entre 5.50 a $7.50 \mathrm{~cm}$ (GÓMEZ, 1997 y ROBLES, 2008).

El rendimiento está influenciado de forma negativa por la incidencia de Alternaria solani, hasta en un $31.74 \%$, en frutos de la producción total por hectárea. No obstante, el ecotipo CPK-3 ha logrado tener bajo porcentaje de incidencia de Alternaria solani en frutos, con $17.60 \%$, incidencia significativa con respecto a los ecotipos RIJ-101, SM y AMG-2, que alcanzaron hasta un $47.31 \%$ de incidencia de Alternaria Solani en los frutos. El bajo rendimiento del ecotipo RIJ-101, con 9.96 tha $^{-1}$ , se debe a que este posee bajo número de frutos por planta y bajo peso de frutos por planta, así como un alto porcentaje de incidencia de Alternaria solani en frutos, con un $47.31 \%$. Este hecho lo podemos atribuir a sus características genéticas, que condicionaron su respuesta frente a las mismas condiciones climáticas y aplicaciones que se hicieron con los demás ecotipos evaluados.

\section{EFECTO DEL RENDIMIENTO EN EL NÚMERO DE FRUTOS Y PESO DE FRUTOS}

De acuerdo a los componentes del rendimiento (Tabla 2), para el carácter de número de frutos y peso de frutos, el ecotipo CPK-3 ocupó el primer lugar con 126.37 frutos/planta y 8.14 k planta- ${ }^{1}$, diferenciándose significativamente de los demás ecotipos. El CPK -3, logró un mayor rendimiento, debido al tamaño intermedio de su fruto, con peso por fruto de $0.06 \mathrm{~kg}$. Asimismo, este ecotipo cuenta con un mayor número de frutos por planta, ya que las plantas que poseen frutos pequeños tienden a tener mayor número de frutos (VILLACHICA, 1996 FLORES 1997).

La influencia del número de frutos por planta en el rendimiento está representada por la línea de regresión simple, con una recta que tiene relación positiva (Figura1) y con un coeficiente de correlación positiva (r) no significativa de 0.494 , en donde al aumentar el número de frutos en una unidad por planta aumentará el rendimiento. Esto indica que por cada unidad de fruto incrementada por planta, el rendimiento también se incrementará en $77.4 \mathrm{~kg} . \mathrm{ha}^{1}$. 
No obstante, este incremento solo simboliza el $24.5 \%$ de influencia, lo que quiere decir que el $75.6 \%$ de rendimiento se debe a otros factores desconocidos.

El efecto del peso de fruto por planta, según la Figura 2, muestra la línea de regresión simple con un coeficiente de correlación positiva (r) altamente significativa, igual a 1.00 , en donde al aumentar el peso de fruto en una unidad por planta, aumentará el rendimiento. Asimismo, presenta un coeficiente de determinación de 0.9996, cuyo valor indica que el 99.96\% de la variación en el rendimiento se debe al peso de frutos por planta en este experimento. La influencia del peso de frutos por planta en el rendimiento está representada por la recta mostrada con una relación positiva perfecta, teniendo como resultado que por cada unidad de peso incrementado por planta, el rendimiento también se incrementara en $3240.7 \mathrm{~kg} \mathrm{ha}^{-1}$, lo que simboliza el $99.96 \%$ de influencia sobre el rendimiento.

\section{INCIDENCIA DE LA ENFERMEDAD CAUSADA POR Alternaria solani}

De los resultados se deduce que para el porcentaje de incidencia de la enfermedad en los frutos de cocona (Tabla 3), el ecotipo CPK-3 alcanzó la más alta resistencia a la enfermedad, con un porcentaje de incidencia del $17.60 \%$, sin embargo, no resultó significativo frente a los ecotipos NE y SH-4, encontrándose diferencias estadísticas frente a los ecotipos AMG-2, SM y RIJ-101. El ecotipo RIJ-101 alcanzó la más baja resistencia a la enfermedad con un porcentaje de incidencia del $47.31 \%$.

La respuesta al ataque de Alternaria solani a los frutos en los diferentes ecotipos ha sido variada, tal como se muestra en la Tabla 3. El ecotipo CPK-3 alcanzó el menor porcentaje de incidencia $(17.60 \%)$ de la enfermedad. Sin embargo, no resultó significativo frente a los ecotipos NE y SH-4, encontrándose diferencias estadísticas frente a los ecotipos AMG-2, SM y RIJ-101. Los ecotipos NE, SH-4, AMG-2, SM y RIJ-101 alcanzaron un porcentaje de incidencia de la enfermedad de $24.99 \% ; 25.77 \%$; $34.16 \% ; \quad 40.62 \%$ y $47.31 \%$ respectivamente.

El efecto del porcentaje de incidencia de Alternaria solani en el rendimiento, según la Figura 3, es mostrado por la línea de regresión simple con un coeficiente de correlación negativa (r) fuerte igual a 0.538 , donde al aumentar el porcentaje de incidencia de $A$. solani en una unidad, disminuirá el rendimiento en $317.80 \mathrm{k}$ planta- ${ }^{1}$. Asimismo, presenta un coeficiente de determinación de 0.2898 , valor que nos indica que el $28.98 \%$ de la variación negativa en el rendimiento se debe al porcentaje de incidencia de enfermedades y que el $71.02 \%$ del rendimiento dependerá de otros factores desconocidos.

\section{CONCLUSIONES}

1. El rendimiento del ecotipo CPK-3 (27.13 $\left.\mathrm{t} \mathrm{ha}^{-1}\right)$ es estadísticamente similar al ecotipo AMG-2 $\left(20.86 \mathrm{t} \mathrm{ha}^{-1}\right)$, sin embargo, el ecotipo CPK-3 es estadísticamente superior a los ecotipos $\mathrm{SM}$ (15.79 $\left.\mathrm{t} \mathrm{ha}^{-1}\right)$, SH-4 (12.99 $\left.\mathrm{t} \mathrm{ha}^{-1}\right)$, NE (11.54 t $\left.\mathrm{ha}^{-1}\right)$ y RIJ-101 (9.97 tha ${ }^{-1}$.

2. En el carácter peso por fruto, el ecotipo SM $(0.18 \mathrm{~kg})$ superó estadísticamente al ecotipo CPK-3 (0.06 kg), mientras que en el carácter número de frutos por planta, el ecotipo CPK-3 (126.37 frutos por plantas) superó estadísticamente al ecotipo SM (26.28 frutos por planta).

3. Los ecotipos que presentaron menor incidencia de Alternaria solani a nivel de frutos fueron: CPK-3 (17.60\%), NE (24.99\%) y $\mathrm{SH}-4$ (25.77\%), mientras que el ecotipo RIJ-101 presentó la mayor incidencia con $47.31 \%$. 


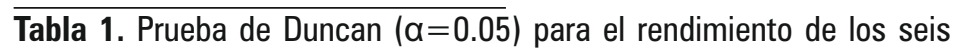
ecotipos de cocona en seis cosechas realizadas.

\begin{tabular}{ccc}
\hline Ecotipo & Rendimiento $\left(\mathbf{t ~ h a}^{-1}\right)$ & Sign. \\
\hline CPK-3 & 27.13 & $\mathrm{a}$ \\
AMG-2 & 20.87 & $\mathrm{a} \mathrm{b}$ \\
SM & 15.79 & $\mathrm{~b} \mathrm{c}$ \\
SH-4 & 13.01 & $\mathrm{C}$ \\
NE & 11.54 & $\mathrm{c}$ \\
RIJ-101 & 9.97 & $\mathrm{c}$ \\
\hline
\end{tabular}

Promedios de tres repeticiones con igual letra no son significativos entre sí. $\alpha=0.05$.

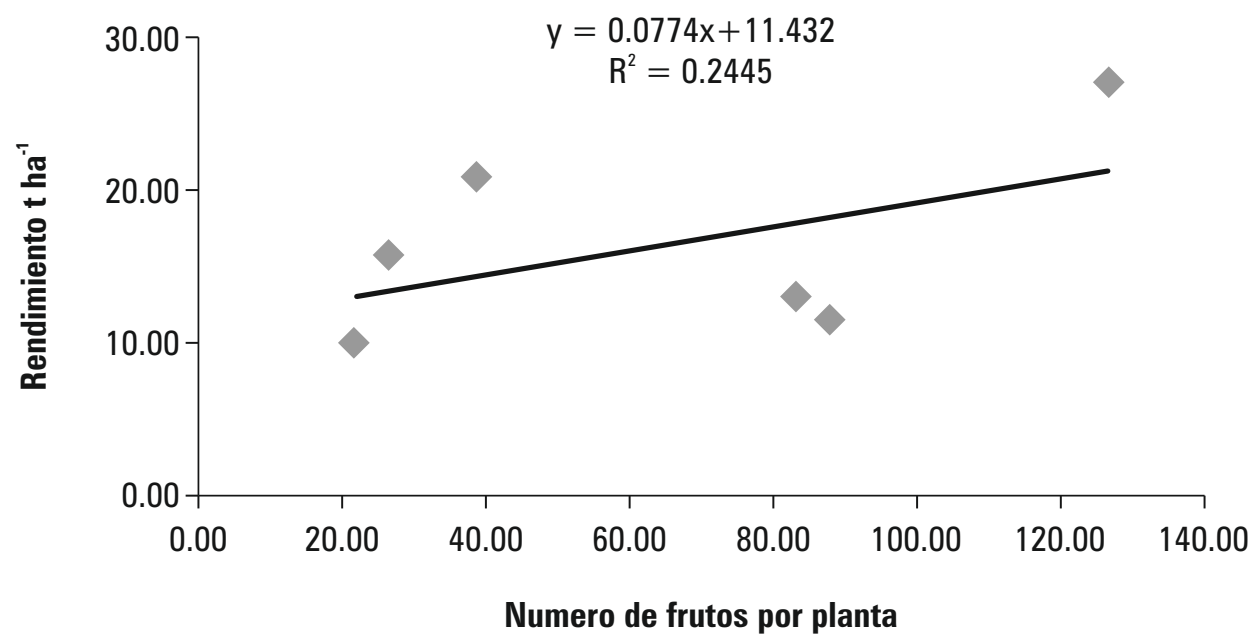

Figura 1. Relación entre el número de frutos por planta y el rendimiento. 
Tabla 2. Prueba de Duncan $(\boldsymbol{\alpha}=0.05)$ para el número de frutos y peso de frutos de seis ecotipos de cocona en seis cosechas.

\begin{tabular}{ccccccccc}
\hline Ecotipo & $\begin{array}{c}\text { Número de } \\
\text { frutos/planta }\end{array}$ & $\begin{array}{c}\text { Número de } \\
\text { frutos/ha. }\end{array}$ & Sign. & Ecotipo & $\begin{array}{c}\text { Peso de } \\
\text { fruto/planta } \\
\text { (kg) }\end{array}$ & $\begin{array}{c}\text { Peso de } \\
\text { fruto } \\
\text { (kg) }\end{array}$ & $\begin{array}{c}\text { Peso de } \\
\text { fruto por } \\
\text { planta/ha } \\
\text { (kg) }\end{array}$ & Sign. \\
\hline CPK-3 & 126.37 & 421191.21 & a & CPK-3 & 8.14 & 0.06 & 27130.62 & a \\
\hline NE & 87.79 & 292604.07 & b & AMG-2 & 6.26 & 0.16 & 20864.58 & a b \\
\hline SH-4 & 83.08 & 276905.64 & b & SM & 4.74 & 0.18 & 15798.42 & b c \\
AMG-2 & 38.76 & 129187.08 & c & SH-4 & 3.90 & 0.05 & 12998.70 & c \\
SM & 26.28 & 87591.24 & C & NE & 3.46 & 0.04 & 11532.18 & c \\
RIJ-101 & 21.82 & 72726.06 & C & RIJ-101 & 2.99 & 0.13 & 9965.67 & C \\
\hline
\end{tabular}

Promedios con igual letra no son significativos entre sí.

Leyenda:

NFP : Número de frutos por planta

NFha : Número de frutos por hectárea

PFP : Peso de frutos por planta

PF : Peso de fruto individual

PFha : Peso de frutos por hectárea

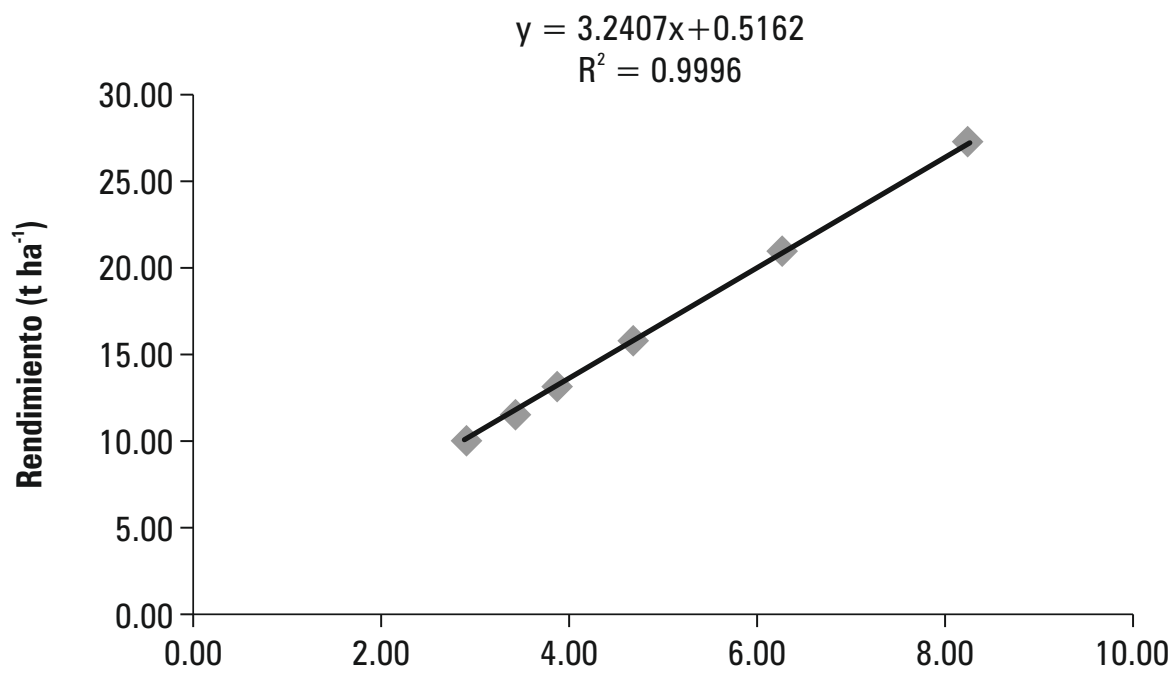

Peso fruto por planta $(g)$

Figura 2. Relación entre el peso de frutos por planta y el rendimiento. 
Tabla 3. Prueba de Duncan $(\alpha=0.05)$ para el porcentaje de incidencia de Alternaria solani en los frutos durante las seis cosechas en los seis ecotipos de cocona.

\begin{tabular}{ccc}
\hline Ecotipo & Rendimiento (\%) & Significación \\
\hline RIJ-101 & 47.31 & a \\
SM & 40.62 & a b \\
AMG-2 & 34.16 & b c \\
SH-4 & 25.77 & c d \\
NE & 25.00 & c d \\
\hline CPK-3 & 17.60 & d \\
\hline
\end{tabular}

Promedios con igual letra no son significativos entre sí.

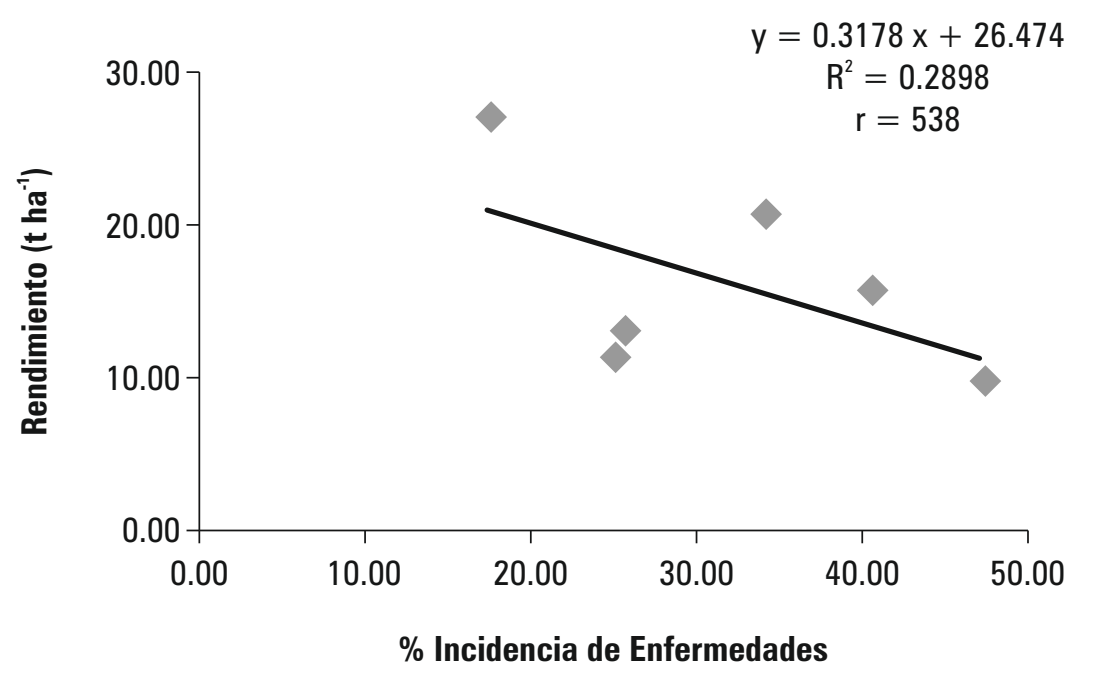

Figura 3. Relación entre el porcentaje de incidencia de Alternaria solani y el Rendimiento. 


\section{BIBLIOGRAFÍA CITADA}

Anculle, A. y Álvarez, R. 1999.Evaluación de enfermedades de plantas. Versión 2.Arequipa ( $\mathrm{P}$ e $\quad \mathrm{r}$ ú ) . (http://es.scribd.com/doc/151857253/Evaluaci on-de-Enfermedades-de-Las-Plantas). Acceso: 24/03/2014.

Carbajal, T.; Balcázar, L. 2000. La cocona. Instituto de la Amazonia Peruana, Tingo María, Perú. 56 p.

Balcázar, T.; Carbajal, C.; Anteparra, M.; Cabezas, O. 2011. El cultivo de Cocona. CONCYTEC, Lima, Perú. $115 \mathrm{p}$.

DiRienzo, C. 2005. Estadística para las ciencias agropecuarias. Edit. ISBN. Córdova. Argentina. $347 \mathrm{p}$.

Flores, S.1997. Cultivo de frutales nativos amazónicos. (Tratado de Cooperación Amazónica - secretaria Pro tempore). Lima Perú. $71-75 \mathrm{p}$.

María, P.S. 2004. Efecto de Solanum sessiliflorum Dunal sobre el metabolismo lípido y la glucosa.
Universidad particular Norbert Wiener. Ciencia e Investigación VII (2). Facultad de Farmacia y Bioquímica. UNMSM. ISSSN 1561-0861. 6 p.

Michel, L.M.; Novisel, V.R.; Miguel D.R. 2001.Evaluación de diferentes somaclones de papa para la resistencia a Alternaria solani Sorauer, Mediante el uso de la inoculación artificial en condiciones de campo. Biotecnología vegetal Vo1 №2:93-96, mayoagosto 2001. 4p.

Robles, R. 2008. Comparativo de rendimiento de cuatro ecotipos de cocona (Solanum sessiliflorum Dunal) en Tingo María. Tesis. Ing. Agrónomo. Universidad Nacional Agraria de la Selva. Tingo María. Perú. 108 p.

Villachica, H. 1996. Frutales y hortalizas promisorios de la Amazonia. (Tratado de cooperación amazónica - secretaria Pro tempore) $N^{\circ} 4$. Lima-Perú. 99-102 p. 